\section{PRC2 directly methylates GATA4 and represses its transcriptional activity}

\author{
Aibin He, ${ }^{1,2}$ Xiaohua Shen, ${ }^{2,3,6}$ Qing Ma, ${ }^{1,2}$ \\ Jingjing Cao, ${ }^{1,2}$ Alexander von Gise, ${ }^{1,2}$ \\ Pingzhu Zhou, ${ }^{1,2}$ Gang Wang, ${ }^{1,2}$ Victor E. Marquez, ${ }^{4}$ \\ Stuart H. Orkin, ${ }^{2,3,5}$ and William T. $\mathrm{Pu}^{1,2,7}$ \\ ${ }^{1}$ Department of Cardiology, Children's Hospital Boston, Harvard \\ Medical School, Boston, Massachusetts 02115, USA; ${ }^{2}$ Harvard \\ Stem Cell Institute, Harvard University, Cambridge, \\ Massachusetts 02138, USA; ${ }^{3}$ Department of Pediatric Oncology, \\ Dana-Farber Cancer Institute, Children's Hospital Boston, \\ Harvard Medical School, Boston, Massachusetts 02115, USA; \\ ${ }^{4}$ Chemical Biology Laboratory, Center for Cancer Research, \\ NCI-Frederick, Frederick, Maryland 21702, USA; ${ }^{5}$ Howard \\ Hughes Medical Institute, Boston, Massachusetts 02115, USA
}

Polycomb-repressive complex 2 (PRC2) promotes tissuespecific differentiation by depositing trimethylated histone H3 Lys 27 (H3K27me3) epigenetic marks to silence ectopic gene expression programs. Here, we show that EZH2, the catalytic subunit of PRC2, is required for cardiac morphogenesis. Both in vitro and in fetal hearts, EZH2 interacted with cardiac transcription factor GATA4 and directly methylated it at Lys 299. PRC2 methylation of GATA4 attenuated its transcriptional activity by reducing its interaction with and acetylation by p300. Our results reveal a new mechanism of PRC2-mediated transcriptional repression in which PRC2 methylates a transcription factor to inhibit its transcriptional activity.

Supplemental material is available for this article.

Received July 7, 2011; revised version accepted November 23, 2011.

Polycomb-repressive complex 2 (PRC2) promotes tissuespecific differentiation by silencing ectopic gene expression programs (for review, see Schuettengruber et al. 2007). PRC2 has been shown to repress gene expression by trimethylating histone H3 Lys 27, establishing repressive epigenetic marks (Cao et al. 2002; Kuzmichev et al. 2002). The PRC2 complex contains the subunits Enhancer of Zeste 2 (EZH2), Embryonic Ectoderm Development (EED), and Suppressor of Zeste 12 (SUZ12) (Kuzmichev et al. 2002). EZH2 is the catalytic subunit that transfers a methyl group from S-adenosyl methionine (SAM) to H3K27 (Cao et al. 2002; Kuzmichev et al. 2002). EED and SUZ12 are required to stabilize EZH2 (Pasini et al. 2004; Montgomery et al. 2005). By establish-

[Keywords: epigenetics; heart development; polycomb complex; transcriptional regulation]

${ }^{6}$ Present address: School of Medicine, Tsinghua University, Beijing 10084, China.

${ }^{7}$ Corresponding author.

E-mail wpu@enders.tch.harvard.edu.

Article is online at http://www.genesdev.org/cgi/doi/10.1101/gad.173930.111. ing trimethylated $\mathrm{H} 3 \mathrm{~K} 27$ (H3K27me3) epigenetic marks that repress inappropriate transcriptional programs, PRC2 executes pluripotency in embryonic stem (ES) cells (Pasini et al. 2007; Shen et al. 2008) and regulates tissue-specific differentiation (Ezhkova et al. 2009; Hirabayashi and Gotoh 2010).

The transcription factor GATA4 is a key, dosage-sensitive regulator of heart development in mice and humans (Garg et al. 2003; Pu et al. 2004; Rajagopal et al. 2007). GATA4 binds p300 (Dai and Markham 2001) and recruits p300 to specific chromatin loci. Furthermore, p300 acetylates GATA4, thereby augmenting its transcriptional activity (Takaya et al. 2008; Trivedi et al. 2010). Here, we show that EZH2 bound and directly methylated GATA4 in the fetal heart. GATA4 methylation by PRC2 impaired its acetylation by p300 and reduced its recruitment of p300 to chromatin, resulting in reduced GATA4 transcriptional potency. This result establishes a new mechanism of PRC2 repression, in which PRC2 directly methylates a transcription factor to attenuate its transcriptional activity.

\section{Results and Discussion}

We previously described a system to express in vivo biotinylated GATA4 (He et al. 2011). In this system, dual adenoviruses express GATA4 bearing Flag and bio epitope tags (Gata4 ${ }^{\text {flbio }}$ ) and BirA, an Escherichia coli enzyme that recognizes and biotinylates the bio substrate peptide (Supplemental Fig. S1A). Transduction of the cardiomyocyte-like HL1 cell line (Claycomb et al. 1998; White et al. 2004) with these adenoviruses led to expression of biotinylated GATA4 at near-endogenous levels (Supplemental Fig. S1B,C). This system permitted high-affinity GATA4 pulldown on streptavidin (SA) beads. To identify transcriptional regulators that interact with and modulate GATA4 activity, we screened candidate factors for GATA4 binding and found that GATA $4^{\text {flbio }}$ specifically coprecipitated the core PRC2 subunits EZH1, EZH2, and SUZ12 (Fig. 1A). The PRC2-associated factor JARID2 (Shen et al. 2009) was also coprecipitated. In contrast, non-PRC2 epigenetic regulators ASH2L, WDR5, and RbBP5 did not coprecipitate and supported specificity of the pull-down (Fig. 1A). Nuclease treatment did not reduce PRC2 coprecipitation, indicating that it was not a consequence of tethering through DNA. The interaction was confirmed by demonstrating GATA $4^{\text {flbio }}$ in EZH2 immunoprecipitates (Fig. 1B).

To further define GATA4-PRC2 interactions, we used in vitro translation to synthesize and ${ }^{35} \mathrm{~S}$-methioninelabel GATA4 $4^{\text {flbio }}$ and three core PRC2 components: EZH2, SUZ12, and EED. When individually incubated with GATA4 ${ }^{\text {flbio }}$, EZH2, but not EED or SUZ12, was recovered in Flag immunoprecipitates (Fig. 1C), indicating that GATA $4{ }^{\text {flbio }}$ interacts with EZH2. GATA4 protein-protein interactions frequently involve its DNA-binding domain. Therefore, we tested interaction of EZH2 with a chemically synthesized, biotinylated peptide $\left(\mathrm{G} 4 \mathrm{P} 1^{\text {bio }}\right)$ corresponding to the minimal GATA4 DNA-binding domain, composed of the C-terminal zinc finger and adjoining basic region (murine GATA4 residues 249-323). G4P1 ${ }^{\text {bio }}$ pulled down in vitro translated EZH2 (Fig. 1D), showing that the minimal GATA4 DNA-binding domain interacts with EZH2 and further supporting direct GATA4 interaction with EZH2. 


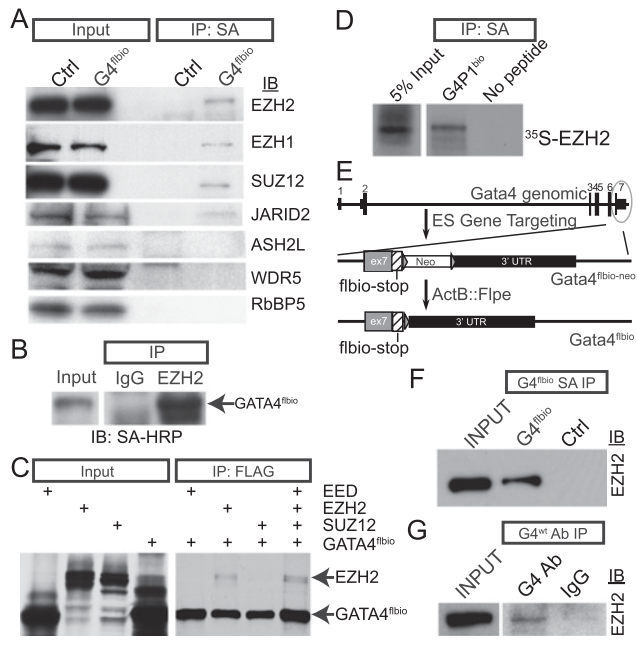

Figure 1. EZH2 interacted with GATA4. (A) GATA4 ${ }^{\mathrm{flbio}}$, expressed in HL1 cells and precipitated on SA beads in the presence of benzonase, coprecipitated PRC2 components EZH2, EZH1, SUZ12, and JARID2. ASH2, WDR5, and RbBP5 were not detected in the precipitate. $|B|$ EZH2, immunoprecipitated from HL1 cells expressing GATA4 4 flbio, coprecipitated GATA4. Biotinylated GATA4 was detected with SAHRP. (C) EED, EZH2, SUZ12, and Flag-tagged GATA4 ${ }^{\text {flbio }}$, synthesized and ${ }^{35} \mathrm{~S}$-labeled in vitro, were Flag-immunoprecipitated. EZH2 specifically interacted with GATA4. (D) Chemically synthesized G4P1 ${ }^{\text {bio }}$ peptide (murine GATA4 residues 249-323) coprecipitated ${ }^{35}$ S-labeled, in vitro translated EZH2 on SA beads. (E) Gene targeting strategy for generation of GATA $4^{\text {flbio }}$ knock-in mice. C-terminal Flag and bio epitope tags were placed on the GATA4 C terminus. $(F)$ Nuclear extracts from GATA $4^{\text {flbio/flbio }}$ Rosa2 $6^{\text {BirA/BirA }}$ or GATA4 $4^{+/+}$ Rosa26 $6^{\text {BirA/BirA }}$ (control) E16.5 embryo hearts were incubated with SA beads. Coprecipitated EZH2 was detected by immunoblotting. $(G)$ Nuclear extract from wild-type E16.5 embryo hearts was incubated with GATA4 antibody or IgG (control). Coprecipitated EZH2 was detected by immunoblotting.

To determine whether GATA4 interacts with EZH2 in vivo, we generated a GATA4 knock-in allele, GATA4 $4^{\text {flbio }}$ which expressed GATA4 protein with the C-terminal Flagbio-epitope tag (Fig. 1E; Supplemental Fig. S1D,E). In Gata4 ${ }^{\text {flbio/fllbio }}$ Rosa2 $6^{\text {BirA/BirA }}$ mice, the Rosa2 $6^{\text {BirA }}$ allele (Driegen et al. 2005) expressed the E. coli biotinylating enzyme BirA, leading to in vivo biotinylation of GATA4 $4^{\text {flbio }}$. GATA $4^{\mathrm{flbio}}$ mice survived normally (Supplemental Fig. S1F) and had no detectable cardiac abnormalities (Supplemental Fig. S1G,H; data not shown), indicating that the C-terminal tag did not substantially alter GATA4 function. Incubation of embryonic day 16.5 (E16.5) GATA4 ${ }^{\text {flbio/flbio }}$ Rosa26 $6^{\mathrm{BirA} / \mathrm{BirA}}$ heart extract with SA beads precipitated GATA $4^{\text {flbio }}$, and immunoblotting demonstrated specific coprecipitation of EZH2(Fig. 1F). To further confirm this interaction, we performed immunoprecipitation of endogenous GATA4 using a specific antibody. EZH2 coprecipitated with GATA4 from wild-type fetal hearts (Fig. 1G), demonstrating interaction between the endogenous GATA4 and EZH2 in fetal hearts.

PRC2 is a methyltransferase whose major reported substrate is histone H3K27 (Cao et al. 2002; Kuzmichev et al. 2002). Other epigenetic modifying enzymes, such as histone acetyltransferases and histone deacetylases, also modify nonhistone substrates, including transcription factors (Boyes et al. 1998; Yang and Seto 2008; Trivedi et al. 2010). Therefore, we tested the hypothesis that PRC2 methylates GATA4. First, we asked whether GATA4 is methylated. In HL1 cells, GATA4 ${ }^{\text {flbio }}$ pull-down followed by immunoblot- ting with a methyl-lysine (meK)-specific antibody demonstrated GATA4 methylation (Fig. 2A). As a negative control, we performed the experiment in parallel using Tbx $5^{\text {flbio }}$ (He et al. 2011) and found no detectable Tbx5 methylation using the meK antibody (Fig. 2A). To confirm GATA4 methylation in vivo, we used SA beads to pull down GATA4 ${ }^{\text {flbio }}$ from embryo hearts and probed the precipitate with the meK antibody. The meK antibody demonstrated robust GATA4 methylation in fetal hearts (Fig. 2B).

To identify the site of GATA4 methylation, we precipitated GATA $4^{\text {flbio }}$ onto SA beads from E16.5 embryo heart extracts under stringent conditions. Mass spectrometry identified monomethylation of Lys 299 (K299) (Fig. 2C). K299 occurred within an MK motif that is commonly found at sites of lysine methylation (Pang et al. 2010). This residue is located within the EZH2-interacting region, in the GATA4 DNA-binding domain near the junction of the C-terminal zinc finger and the adjacent basic domain.

To determine whether GATA4 methylation requires PRC2, we used shRNA-expressing adenovirus to knock down PRC2 in HL1 cells and assessed the effect on GATA4 methylation. SUZ12 and EED are required to stabilize EZH2 (Montgomery et al. 2005; Pasini et al. 2007). Suz12 depletion ( $>95 \%$ reduction) resulted in depletion of EZH2(Supplemental Fig. S2A). Suz12 shRNA, but not a scrambled shRNA, markedly reduced GATA4 methylation in HLl cells (Fig. 3A). To exclude off-target effects, we used a second shRNA-expressing adenovirus against Eed to deplete PRC2. This shRNA reduced Eed levels (Supplemental Fig. S2B), although it was less efficient than the Suz12 shRNA. EED knockdown also significantly

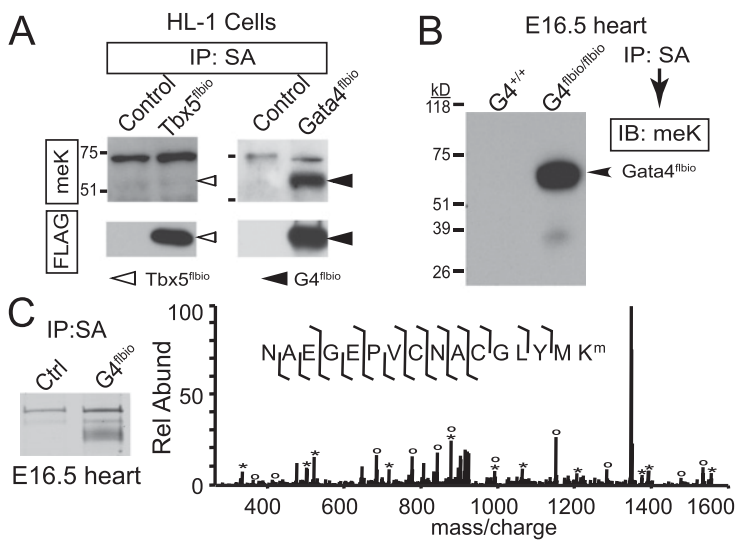

Figure 2. GATA4-K299 was methylated in vivo. (A) GATA4 was methylated in HL1 cells. GATA4 ${ }^{\text {flbio }}$, precipitated from GATA4 $4^{\text {flbio }}$ expressing or control HL1 extracts in the presence of $2 \%$ SDS, immunoreacted with meK-specific antibody at the appropriate molecular weight for GATA4 ${ }^{\text {llbio }}$. No meK immunoreactivity was detected in parallel control experiments with $\mathrm{Tbx} 5^{\mathrm{flbio}}$. (B) GATA4 methylation in E16.5 hearts. Protein lysates from Gata4 $4^{\text {flbio/flbio }}$ Rosa26 $6^{\mathrm{BirA} / \mathrm{BirA}}$ or $\mathrm{Gata}^{+/+}$Rosa26 $6^{\text {BirA/BirA }}$ (control) hearts were analyzed as in $A$. (C) Identification of GATA4 methylation site. GATA4 $^{\text {flbio/flbio }}$ Rosa26 $^{\text {BirA/BirA }}$ or control E16.5 heart protein lysates were precipitated as in $A$. (Inset) SDS-PAGE of precipitated proteins confirmed GATA4 pull-down (colloidal blue-stained). Tandem mass spectrometry identified GATA4 methylation at K299. The mass spectrum of one diagnostic peptide is shown. Fragments containing the original $\mathrm{N}$-terminal amino acid (marked by asterisks) did not show mass shift from a methyl residue, while fragments containing the original C-terminal amino acid (marked by open circles) were shifted by $14 \mathrm{Da}$. Lines in the peptide sequence indicate observed $\mathrm{N}$-terminal and C-terminal fragment ions. 


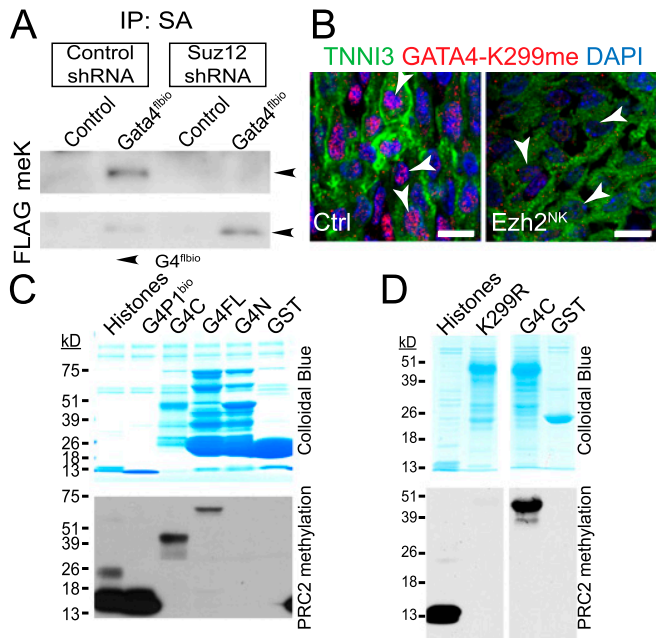

Figure 3. PRC2 directly methylated GATA4. (A) Suz12 shRNA knockdown inhibited methylation of GATA4 ${ }^{\text {lbio }}$ in HL1 cells. GATA4 ${ }^{\text {flbio }}$ methylation was assessed as in Figure 2, A and B. The arrowhead indicates the position of the GATA $4^{\text {flbio }}$ band. (B) E16.5 heart cryosections stained for cardiac marker TNNI3 and K299. methylated GATA4. GATA4-K299me immunoreactivity (arrowheads) was markedly reduced in Ezh2 $2^{\mathrm{NK}}$ mutant cardiomyocytes. (C) Recombinant PRC2 methylated GATA4 fragments that encompass K299. GATA4 was chemically synthesized $\left(\mathrm{G} 4 \mathrm{P} 1^{\text {bio }}\right.$, residues 249-323) or purified as GST fusion proteins from bacteria. GATA4 residues in the constructs are G4P1 ${ }^{\text {bio }}, 249-323$; G4C, 207-441; G4FL, 1-441; and G4N, 1-214. After incubation with PRC2 and ${ }^{3} \mathrm{H}$-SAM, reaction products were resolved by SDS-PAGE and detected by autoradiography. Histones were used as positive control. (D) Mutation of GATA4-K299 abrogated methylation by PRC2.

reduced GATA4 methylation (Supplemental Fig. S2C), albeit less completely than Suz12 shRNA. Collectively, these data indicated that PRC2 is required for GATA4 methylation in HL1 cells.

To determine whether PRC2 is required to methylate GATA4 in vivo, we assessed GATA4 methylation in mouse embryo hearts in which a conditional allele of Ezh2 (Ezh2 ${ }^{\mathrm{fl}}$ ) (Shen et al. 2008) was inactivated in the heart by Nkx2-5 Cre (Moses et al. 2001). By E12.5, Ezh2 was efficiently inactivated in cardiomyocytes (Supplemental Fig. S3A,B). We developed a polyclonal antibody that specifically recognized GATA4 monomethylated at K299, but not unmethylated GATA4 (Supplemental Fig. S3C). While GATA4 expression and immunoreactivity were unchanged in Ezh2 ${ }^{\mathrm{NK}}$ mutants (Supplemental Fig. S3D,E), GATA4-K299me immunoreactivity was markedly reduced (Fig. 3B): GATA4K299me immunoreactivity was detected in nearly all control cardiomyocytes, but we detected it in only $4.2 \% \pm$ $1.0 \%$ of Ezh2 ${ }^{\mathrm{NK}}$ mutant cardiomyocytes $(n=3)$. These data indicate that EZH2 is required to methylate GATA4 in fetal hearts.

To test the hypothesis that PRC2 directly methylates GATA4, recombinant, purified PRC2 (Shen et al. 2008) was incubated with purified bacterially expressed proteins consisting of regions of GATA4 fused to a GST affinity tag. PRC2 methylated GST-GATA4[1-441] (G4 FL) and GSTGATA4[207-441] (G4C), but not GST alone or GSTGATA4[1-244] (G4N) (Fig. 3C). Bacterial proteins also present in the GST fusion protein preparations were not methylated by PRC2, demonstrating PRC2 substrate specificity. The G4P1 ${ }^{\text {bio }}$ peptide also was robustly methylated by PRC2 (Fig. 3C). These data indicate that PRC2 directly methyl- ated GATA4 between residues 249-323. This result is consistent with in vivo GATA4 methylation at K299, as shown by mass spectroscopy. We confirmed PRC2 methylation of this residue in the methyltransferase assay by using site-directed mutagenesis to obtain GST-G4C[K299R]. PRC2 failed to methylate this mutant protein (Fig. 3D). This was not due to gross disruption of GATA4 structure, because the mutant protein retained EZH2- and DNAbinding activity (Supplemental Fig. S4A,B). Collectively, these data clearly indicate that PRC2 methylates GATA4 at K299, demonstrating for the first time that PRC2 methylation extends to nonhistone proteins, including transcription factors.

Consistent with an essential role of Ezh2 in heart development, Ezh2 ${ }^{\mathrm{NK}}$ embryos died perinatally, with thinning of the compact myocardium and hypertrabeculation (Supplemental Fig. S5A,B). A detailed description of these mutant embryos will be reported elsewhere. To evaluate the functional effect of GATA4 methylation by PRC2, we tested GATA4 target genes for differential expression in Ezh2 ${ }^{\text {NK }}$ embryo heart ventricles. GATA4 regulates expression of the essential sarcomere gene Myh6, which encodes myosin heavy chain $\alpha$ (Zeisberg et al. 2005). PRC2 repressed Myh6, as Myh6 expression was up-regulated in Ezh2 ${ }^{\text {NK }}$ heart ventricles (Fig. 4A). This was recapitulated in both cultured neonatal rat ventricular cardiomyocytes (NRVMs) and HL1 cells depleted of PRC2 by treatment with the small molecule DZNep (Tan et al. 2007; Fiskus et al. 2009) or Suz12 shRNA (Fig. 4B,C; Supplemental Fig. S6). Although PRC2 repressed Myh6 in multiple contexts, neither EZH2 nor H3K27me3 was substantially enriched in wild-type hearts at proximal Myh6 regulatory sequences (Supplemental Fig. S7), which are sufficient to drive cardiomyocyte-specific gene expression (Gulick et al. 1991). H3K27me3 repressive marks are broadly distributed and enriched near the transcriptional start site of repressed genes (Barski et al. 2007; Young et al. 2011). Thus, the established mechanism of PRC2 repression involving H3K27me3 deposition at target gene promoters was unlikely to account for its repression of Myh6.

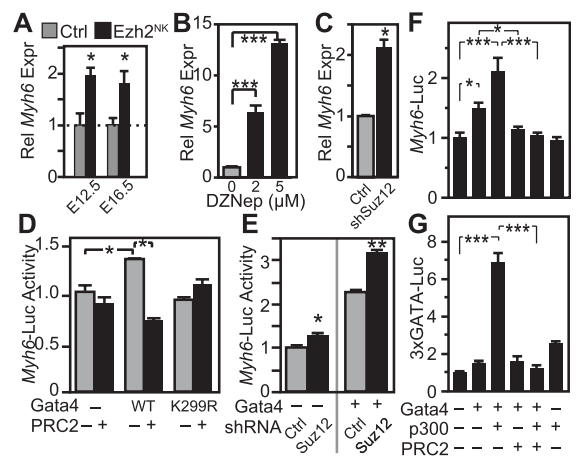

Figure 4. PRC2 modulated GATA4 activation of Myh6. (A) Myh6 transcripts were up-regulated in EZH2-deficient E12.5 and E16.5 fetal hearts. (B) PRC2 knockdown by DZNep up-regulated endogenous Myh6 transcript in NRVMs. (C) Suz12 knockdown in HL1 upregulated the endogenous Myh6 transcript. (D) GATA4 activation of Myh6-luc was blocked by transfection of PRC2 components Ezh2, Eed, and Suz12 and by K299R mutation. (E) Suz12 knockdown in HL1 up-regulated activity of Myh6-luc and potentiated the effect of GATA4 overexpression. $(F, G)$ PRC2 abolished p300 stimulation of GATA4 transcriptional activity on Myh6-luc and 3xGATA-luc reporters. $\left.\left(^{\star}\right) P<0.05 ;\left(^{\star \star}\right) P<0.01 ;\left.\right|^{\star \star \star}\right) P<0.001$. 
Myh6 regulatory sequences were occupied by GATA4 (Supplemental Fig. S7). We therefore hypothesized that PRC2 regulates Myh6 by attenuating the transcriptional activity of GATA4, which is required to activate Myh6 (Zeisberg et al. 2005). The G4P1me ${ }^{\text {bio }}$ peptide bound DNA with reduced affinity compared with the unmethylated peptide-bound DNA (Supplemental Fig. S8A). However, PRC2 knockdown in HL1 cells did not alter GATA4 occupancy of Myh6 regulatory elements in HL1 cells (Supplemental Fig. S8B) or six additional GATA4-bound loci examined, identified by GATA4 chromatin immunoprecipitation (ChIP) and sequencing (ChIP-seq) in this cell line (Supplemental Fig. S8B,C; He et al. 2011). Thus, PRC2 does not appear to influence GATA4 chromatin occupancy, and this mechanism is unlikely to account for the effect of PRC2 on Myh6 expression. Therefore, we used a Myh6 luciferase reporter containing the $5.5-\mathrm{kb}$ Myh6 promoter/enhancer (Gulick et al. 1991) and the GATA4-binding site to ask whether GATA4 methylation alters its transcriptional activity. In transfected HL1 cells, the Myh6-luc reporter was activated by GATA4 overexpression, and this up-regulation was blocked by overexpression of PRC2 (Fig. 4D). Western blotting for GATA4 showed that PRC2 overexpression did not inhibit reporter activity by decreasing GATA4 levels (data not shown). Conversely, PRC2 knockdown by Suz12 shRNA abolished GATA4 methylation (Fig. 3A) and strongly augmented GATA4 activation of Myh6-luc (Fig. 4E). These results indicate that PRC2 inhibited GATA4 transcriptional activity and that this inhibition occurs even in a transfected reporter construct without the chromatin structure of genomic DNA.

The transcriptional coactivator p300 interacts with GATA4 and enhances its transcriptional activity by acetylating lysine residues in its C-terminal region (Takaya et al. 2008; Trivedi et al. 2010). Lysines at positions 311, 318,320 , and 322 , but not 299, were identified as the major sites of p300-mediated GATA4 acetylation (Takaya et al. 2008). Consistent with prior studies, cotransfection of p300 augmented GATA4 transcriptional activity on Myh6-luc (Fig. 4F). p300-stimulated GATA4 transcriptional activity was even more robust on a second reporter composed of three GATA4-binding sites upstream of a minimal promoter and luciferase (Fig. 4G; Durocher et al. 1997). Remarkably, p300-stimulated GATA4 transcriptional activity on both reporters was abolished by cotransfection of PRC2 (Fig. 4F,G). This result suggested that PRC2 methylation of GATA4 inhibited its acetylation by p300.

We tested the hypothesis that PRC2 methylation of GATA4 inhibits its acetylation by p300 using an in vitro p300 acetyltransferase assay. Consistent with previous results (Takaya et al. 2008), p300 acetylated the C-terminal region of GATA4 in vitro (Fig. 5A). Methylation of GATA4 by PRC2 preincubation significantly inhibited GATA4 acetylation $\left(\left[{ }^{\star}\right] P<0.05\right)$ (Fig. 5A,B). Since in vitro PRC2 methylation is not quantitative, the approximately twofold reduction of GATA4 acetylation we observed likely underestimated the PRC2 inhibitory effect. The inhibitory effect of PRC2 required the methyl group donor SAM, indicating that the inhibitory effect of PRC2 requires GATA4 methylation. To further investigate the mechanism by which PRC2 impaired GATA4 acetylation by p300, we measured the binding of p300 to GST-GATA4[207-441] with mock or PRC2 preincubation. Preincubation of GATA4 with PRC2 reduced p300 binding (Supplemental Fig. S9A).

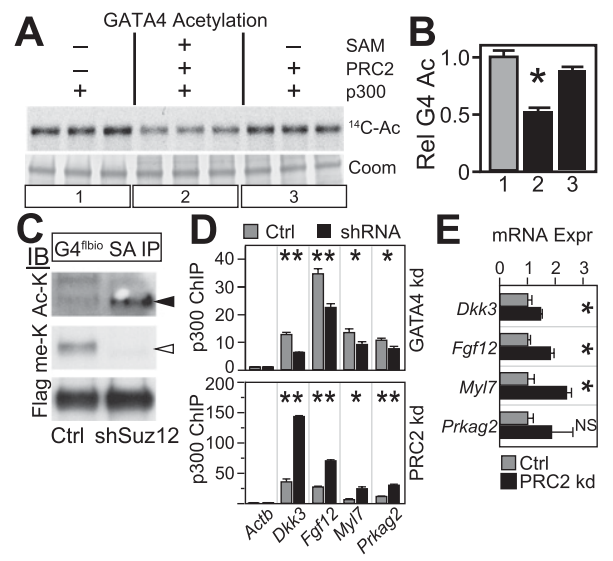

Figure 5. PRC2 antagonized $\mathrm{p} 300$ acetylation of and binding to GATA4 in vitro and in vivo. (A) In vitro GATA4 acetylation by p300 was markedly attenuated by preincubation with PRC2 and the methyl donor SAM. Acetylation of GST fusion proteins was detected by p300-mediated labeling by ${ }^{14} \mathrm{C}$-acetyl-CoA. Coom indicates Coomassie blue-stained gel, which showed equal protein loading. $(B)$ Quantitation of $A$. Numbers correspond to groups indicated in $A .(C)$ PRC2 regulates GATA4 acetylation in HL1 cells. GATA4 ${ }^{\text {flbio }}$-expressing HL1 cells were treated with control or Suz12 shRNA. GATA4 $4^{\text {flbio }}$ was then pulled down and probed with acetyl lysine or meK antibodies. (Open arrowhead) Methylated GATA4; (closed arrowhead) acetylated GATA4. (D) PRC2 regulates p300 recruitment by GATA4 in HL1 cells. p300 occupancy at indicated loci was determined by ChIP-qPCR in the context of GATA4 or PRC2 knockdown. (E) Effect of PRC2 on mRNA expression in HL1, as measured by qRT-PCR. $\left(^{*}\right) P<0.05$; $\left(^{\star \star}\right) P<0.01$; (NS) not significant.

This effect was due to GATA4 methylation rather than competition between EZH2 and p300 for overlapping GATA4binding sites, because preincubation of the GATA4[K299R] mutant with PRC2 had no effect on p300 binding (Supplemental Fig. S9A). This mutant binds to both p300 (Supplemental Fig. S9A) and EZH2 (Supplemental Fig. S4A) but cannot be methylated. To determine whether PRC2 influences GATA4 acetylation in cardiomyocytes, we depleted PRC2 in HL1 cells using shSuz12 adenovirus and measured the effect on GATA4 acetylation. Consistent with the in vitro data, PRC2 depletion strongly increased GATA4 acetylation in HL1 cells (Fig. 5C). Taken together, the data indicate that PRC2 represses GATA4 transcriptional activity by methylating K299 and inhibiting its binding to and acetylation by $\mathrm{p} 300$.

In cardiomyocytes, GATA4 is a major determinant of p300 chromatin occupancy, as $76 \%$ of p300-occupied loci overlap with GATA4-occupied loci in HL1 cells (He et al. 2011). This suggested the hypothesis that PRC2 antagonizes GATA4 recruitment of p300 to chromatin loci. To test this hypothesis, we first identified loci at which p300 recruitment was GATA4-dependent. Based on GATA4 and p300 chromatin occupancy data from HL1 (He et al. 2011), we selected loci with co-occurrence of these two factors. GATA4 knockdown in HL1 significantly reduced p300 occupancy at 12 of 21 of these loci (Fig. 5D, top panel; Supplemental Fig. S9B). Of the remaining loci, eight were not significantly changed, and only one showed increased p300 occupancy. Next, we asked whether PRC2 depletion enhanced p300 recruitment to these loci. Indeed, PRC2 knockdown with Suz12 shRNA adenovirus significantly increased p300 recruitment to four of four GATA4-dependent loci tested (Fig. 5D, bottom panel). This effect was 
not mediated by altered GATA4 chromatin occupancy, as demonstrated by quantitative ChIP-qPCR (Supplemental Fig. S8C). Increased p300 recruitment at GATA4-dependent loci was associated with up-regulation of associated gene transcripts, which reached statistical significance in three of four tested genes (Fig. 5E). Together, these data indicate that PRC2 methylation of GATA4 attenuates its recruitment of p300 to chromatin. Furthermore, these data identify several additional target genes whose expression is regulated by PRC2-GATA4 interaction.

Our study shows that GATA4 transcriptional activity is regulated by post-translational modifications of its C-terminal region. We demonstrate that p300 acetylation (Takaya et al. 2008) and PRC2 methylation modify the GATA4 C terminus to stimulate or reduce GATA4 transcriptional activity, respectively (Supplemental Fig. 10). The GATA4 C terminus does not stimulate transcription on its own, but is required for GATA4 transcriptional activity (Morrisey et al. 1997). Thus, the C-terminal domain integrates activating p300 and repressive PRC2 signals to modulate GATA4 transcriptional activity. Furthermore, our data indicate a critical role for residue K299, methylation of which impaired p300 binding, GATA4 acetylation, and GATA4 transcriptional activity. In fact, while the K299R mutation did not impair EZH2, p300, or DNA binding (Supplemental Figs. S4, S9A), it abolished GATA4 methylation (Fig. 3D), p300-mediated acetylation (Supplemental Fig. S9C), and transcriptional activation (Fig. 4D). That the K299R mutant impaired rather than augmented GATA4 transcriptional activity points to the complex role this residue plays in regulating GATA4 activity. Collectively, these data indicate that the C-terminal domain regulates GATA4 transcriptional activity and that this regulation is sensitive to the structure of the residue at position 299.

PRC2 is a critical repressor of inappropriate gene expression programs and is required for normal lineagespecific differentiation and organogenesis (Bracken et al. 2006; Rajagopal et al. 2007; Shen et al. 2008; Ezhkova et al. 2009). PRC2 repressive activity has been attributed to PRC2 trimethylation of histone H3K27. Here, we demonstrate that PRC2 binds and methylates the cardiac transcription factor GATA4, resulting in attenuation of GATA4 transcriptional activity (Supplemental Fig. 10). These findings show for the first time that PRC2 methylates a nonhistone target and establish reduction of methylated transcription factor activity as a new mechanism for PRC2-mediated gene repression. This novel mechanism may be broadly relevant for PRC2regulated gene expression in development and disease.

\section{Materials and methods}

Please see the Supplemental Material for detailed information.

\section{Mice}

Ezh2 ${ }^{\mathrm{fl}}, \mathrm{Nkx2} 25^{\mathrm{Cre}}$, and Rosa2 $6^{\mathrm{BirA}}$ alleles were described previously (Moses et al. 2001; Driegen et al. 2005; Shen et al. 2008). Gata $4^{\text {flbio }}$ knock-in mice were generated by homologous recombination. Experiments were performed according to protocols approved by the Institutional Animal Care and Use Committee.

\section{Gene expression}

qRT-PCR was performed on total RNA isolated, and results were expressed as mean \pm SEM. Primers are listed in Supplemental Table S1.
Protein pull-down and ChIP

ChIP and biotin-mediated ChIP were performed as described (He and $\mathrm{Pu}$ 2010) and expressed as mean \pm SD. Coimmunoprecipitation from tissue was performed using the nuclear extracts and antibodies indicated in the Supplemental Material. In vitro translated proteins were synthesized using the TNT Coupled Reticulocyte Lysate system (Promega).

\section{Methyltransferase and acetyltransferase assays}

In vitro methyltransferase assays were performed with purified PRC2 as described (Shen et al. 2008). Acetyltransferase assays were used with recombinant p300 (Millipore).

\section{Acknowledgments}

This work was supported by funding from the National Institutes of Health (NIH) (U01HL098166 and R01HL095712 to W.T.P.) and the American Heart Association (post-doctoral fellowship to A.H.); by charitable donations from Edward Marram, Karen Carpenter, and Gail Federici Smith; and by a Center of Excellence Award in Molecular Hematology from the NIDDK to S.H.O., an Investigator of the HHMI. This research was supported in part by the Intramural Research Program of the NIH, National Cancer Institute, and Center for Cancer Research. Mass spectrometry analysis was performed by the Proteomics Center at Children's Hospital Boston.

\section{References}

Barski A, Cuddapah S, Cui K, Roh TY, Schones DE, Wang Z, Wei G, Chepelev I, Zhao K. 2007. High-resolution profiling of histone methylations in the human genome. Cell 129: 823-837.

Boyes J, Byfield P, Nakatani Y, Ogryzko V. 1998. Regulation of activity of the transcription factor GATA-1 by acetylation. Nature 396: 594-598.

Bracken AP, Dietrich N, Pasini D, Hansen KH, Helin K. 2006. Genomewide mapping of Polycomb target genes unravels their roles in cell fate transitions. Genes Dev 20: 1123-1136.

Cao R, Wang L, Wang H, Xia L, Erdjument-Bromage H, Tempst P, Jones RS, Zhang Y. 2002. Role of histone H3 lysine 27 methylation in Polycomb-group silencing. Science 298: 1039-1043.

Claycomb WC, Lanson NAJ, Stallworth BS, Egeland DB, Delcarpio JB, Bahinski A, Izzo NJJ. 1998. HL-1 cells: A cardiac muscle cell line that contracts and retains phenotypic characteristics of the adult cardiomyocyte. Proc Nat1 Acad Sci 95: 2979-2984.

Dai YS, Markham BE. 2001. p300 functions as a coactivator of transcription factor GATA-4. J Biol Chem 276: 37178-37185.

Driegen S, Ferreira R, van Zon A, Strouboulis J, Jaegle M, Grosveld F, Philipsen S, Meijer D. 2005. A generic tool for biotinylation of tagged proteins in transgenic mice. Transgenic Res 14: 477-482.

Durocher D, Charron F, Warren R, Schwartz RJ, Nemer M. 1997. The cardiac transcription factors $\mathrm{Nkx} 2-5$ and GATA-4 are mutual cofactors. EMBO J 16: 5687-5696.

Ezhkova E, Pasolli HA, Parker JS, Stokes N, Su IH, Hannon G, Tarakhovsky A, Fuchs E. 2009. Ezh2 orchestrates gene expression for the stepwise differentiation of tissue-specific stem cells. Cell 136: 1122-1135.

Fiskus W, Wang Y, Sreekumar A, Buckley KM, Shi H, Jillella A, Ustun C, Rao R, Fernandez P, Chen J, et al. 2009. Combined epigenetic therapy with the histone methyltransferase EZH2 inhibitor 3-deazaneplanocin A and the histone deacetylase inhibitor panobinostat against human AML cells. Blood 114: 2733-2743.

Garg V, Kathiriya IS, Barnes R, Schluterman MK, King IN, Butler CA, Rothrock CR, Eapen RS, Hirayama-Yamada K, Joo K, et al. 2003. GATA4 mutations cause human congenital heart defects and reveal an interaction with TBX5. Nature 424: 443-447.

Gulick J, Subramaniam A, Neumann J, Robbins J. 1991. Isolation and characterization of the mouse cardiac myosin heavy chain genes. J Biol Chem 266: 9180-9185.

He A, Pu WT. 2010. Genome-wide location analysis by pull down of in vivo biotinylated transcription factors. Curr Protoc Mol Biol 92: 21.20.1-21.20.15. doi: 10.1002/0471142727.mb2120s92.

He A, Kong SW, Ma Q, Pu WT. 2011. Co-occupancy by multiple cardiac transcription factors identifies transcriptional enhancers active in heart. Proc Natl Acad Sci 108: 5632-5637. 
He et al.

Hirabayashi Y, Gotoh Y. 2010. Epigenetic control of neural precursor cell fate during development. Nat Rev Neurosci 11: 377-388.

Kuzmichev A, Nishioka K, Erdjument-Bromage H, Tempst P, Reinberg D. 2002. Histone methyltransferase activity associated with a human multiprotein complex containing the Enhancer of Zeste protein. Genes Dev 16: 2893-2905.

Montgomery ND, Yee D, Chen A, Kalantry S, Chamberlain SJ, Otte AP Magnuson T. 2005. The murine polycomb group protein Eed is required for global histone H3 lysine-27 methylation. Curr Biol 15: 942-947.

Morrisey EE, Ip HS, Tang Z, Parmacek MS. 1997. GATA-4 activates transcription via two novel domains that are conserved within the GATA-4/5/6 subfamily. I Biol Chem 272: 8515-8524.

Moses KA, DeMayo F, Braun RM, Reecy JL, Schwartz RJ. 2001. Embryonic expression of an Nkx2-5/Cre gene using ROSA26 reporter mice. Genesis 31: 176-180.

Pang CN, Gasteiger E, Wilkins MR. 2010. Identification of arginine- and lysine-methylation in the proteome of Saccharomyces cerevisiae and its functional implications. BMC Genomics 11: 92. doi: 10.1186/ 1471-2164-11-92.

Pasini D, Bracken AP, Jensen MR, Lazzerini Denchi E, Helin K. 2004 Suz12 is essential for mouse development and for EZH2 histone methyltransferase activity. EMBO J 23: 4061-4071.

Pasini D, Bracken AP, Hansen JB, Capillo M, Helin K. 2007. The polycomb group protein Suz12 is required for embryonic stem cell differentiation. Mol Cell Biol 27: 3769-3779.

$\mathrm{Pu}$ WT, Ishiwata T, Juraszek AL, Ma Q, Izumo S. 2004. GATA4 is a dosage-sensitive regulator of cardiac morphogenesis. Dev Biol 275: 235-244.

Rajagopal SK, Ma Q, Obler D, Shen J, Manichaikul A, Tomita-Mitchell A, Boardman K, Briggs C, Garg V, Srivastava D, et al. 2007. Spectrum of heart disease associated with murine and human GATA4 mutation. J Mol Cell Cardiol 43: 677-685.

Schuettengruber B, Chourrout D, Vervoort M, Leblanc B, Cavalli G. 2007. Genome regulation by Polycomb and Trithorax proteins. Cell 128: 735-745.

Shen X, Liu Y, Hsu YJ, Fujiwara Y, Kim J, Mao X, Yuan GC, Orkin SH 2008. EZH1 mediates methylation on histone H3 lysine 27 and complements EZH2 in maintaining stem cell identity and executing pluripotency. Mol Cell 32: 491-502.

Shen X, Kim W, Fujiwara Y, Simon MD, Liu Y, Mysliwiec MR, Yuan GC, Lee Y, Orkin SH. 2009. Jumonji modulates polycomb activity and self-renewal versus differentiation of stem cells. Cell 139: 1303-1314

Takaya T, Kawamura T, Morimoto T, Ono K, Kita T, Shimatsu A, Hasegawa K. 2008. Identification of p300-targeted acetylated residues in GATA4 during hypertrophic responses in cardiac myocytes. J Biol Chem 283: 9828-9835.

Tan J, Yang X, Zhuang L, Jiang X, Chen W, Lee PL, Karuturi RK, Tan PB, Liu ET, Yu Q. 2007. Pharmacologic disruption of Polycomb-repressive complex 2-mediated gene repression selectively induces apoptosis in cancer cells. Genes Dev 21: 1050-1063.

Trivedi CM, Zhu W, Wang Q, Jia C, Kee HJ, Li L, Hannenhalli S, Epstein JA 2010. Hopx and Hdac2 interact to modulate Gata4 acetylation and embryonic cardiac myocyte proliferation. Dev Cell 19: 450-459.

White SM, Constantin PE, Claycomb WC. 2004. Cardiac physiology at the cellular level: Use of cultured HL-1 cardiomyocytes for studies of cardiac muscle cell structure and function. Am J Physiol Heart Circ Physiol 286: H823-H829. doi: 10.1152/ajpheart.00986.2003.

Yang XJ, Seto E. 2008. Lysine acetylation: Codified crosstalk with other posttranslational modifications. Mol Cell 31: 449-461.

Young MD, Willson TA, Wakefield MJ, Trounson E, Hilton DJ, Blewitt ME, Oshlack A, Majewski IJ. 2011. ChIP-seq analysis reveals distinct H3K27me3 profiles that correlate with transcriptional activity. Nucleic Acids Res 39: 7415-7427.

Zeisberg EM, Ma Q, Juraszek AL, Moses K, Schwartz RJ, Izumo S, Pu WT. 2005. Morphogenesis of the right ventricle requires myocardial expression of Gata4. J Clin Invest 115: 1522-1531. 


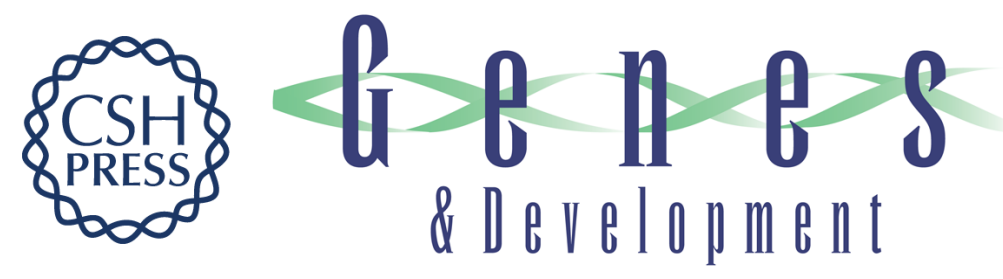

\section{PRC2 directly methylates GATA4 and represses its transcriptional activity}

Aibin He, Xiaohua Shen, Qing Ma, et al.

Genes Dev. 2012, 26:

Access the most recent version at doi:10.1101/gad.173930.111

Supplemental http://genesdev.cshlp.org/content/suppl/2012/01/03/26.1.37.DC1
Material

References This article cites 34 articles, 14 of which can be accessed free at: http://genesdev.cshlp.org/content/26/1/37.full.html\#ref-list-1

\section{License}

Email Alerting

Service

Receive free email alerts when new articles cite this article - sign up in the box at the top right corner of the article or click here.

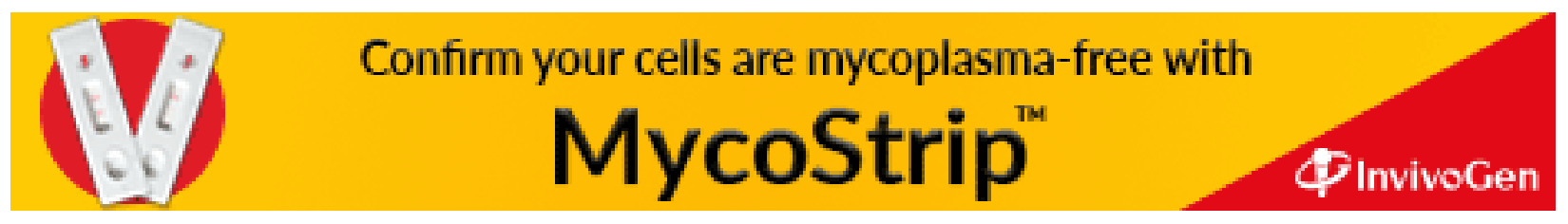

